\title{
ULTRA-STRONG DITKIN SETS IN HYPERGROUPS
}

\author{
AJIT KAUR CHILANA AND AJAY KUMAR
}

\begin{abstract}
We introduce compactly separating sets for hypergroups which under certain conditions turn out to be ultra-strong Ditkin for the hypergroup algebra whenever their boundary is. We also characterize such sets for the hypergroup related to $p$-adic numbers.
\end{abstract}

1. Introduction. Wik [13] defined and studied strong Ditkin sets in the circle group $T$ as those spectral sets $E$ in $T$ for which there exists an approximate identity in the kernel $k(E)$ of $E$ in $l^{1}(Z)$. Rosenthal [9] carried this study further to some locally compact abelian groups $\Gamma$, in particular, he proved that every closed coset in $\Gamma$ is strong Ditkin and a nowhere dense strong Ditkin set is a member of the discrete coset ring $R\left(\Gamma_{d}\right)$. Gilbert [5] proved that closed sets in $R\left(\Gamma_{d}\right)$ are Calderon and Schreiber [10] showed that every such set is strong Ditkin thus completing the characterization of strong Ditkin sets with empty interior. Liu, Rooij and Wang [8] showed that a closed ideal $I$ in $L^{1}(G)$ has a bounded approximate identity if and only if $I$ is the kernel of a closed element $E$ of $R\left(\Gamma_{d}\right)$ where $\Gamma$ is the dual group of $G$. Rosenthal [9] proved that if both $G$ and $\Gamma$ are metrizable then $E$ is strong Ditkin whenever the boundary $\mathrm{Bd} E$ of $E$ is. However his method does not force $E$ to be ultra-strong Ditkin even if $\mathrm{Bd} E$ is. On the contrary, it follows from the above discussion that every proper closed interval $I$ in the additive group $\mathbf{R}$ is strong Ditkin because its boundary is ultra-strong Ditkin whereas $I$ cannot be ultra-strong Ditkin. A study of such notions for hypergroup algebras was begun in [1], [2]. Let $K$ be a locally compact commutative hypergroup with Haar measure $m$ [3], [7], [11], [12] whose dual $\hat{K}$ is a hypergroup and equals the set $\chi_{b}(K)$ of bounded continuous characters on $K, L^{1}(m)=L^{1}(K)$ the convolution algebra and $A(\hat{K})$ the algebra of Fourier transforms $\hat{f}$ of $f$ in $L^{1}(K)$. As in [1], a closed subset $E$ of $\hat{K}$ will be called strong Ditkin if there exists a net $\left\{f_{\alpha}: \alpha \in D\right\}$ in $L^{1}(K)$ such that

(i) for each $\alpha, \hat{f}_{\alpha}=0$ in a neighborhood of $E$ and has compact support,

(ii) $\sup \left\{\left\|f_{\alpha}\right\|_{E}: \alpha \in D\right\}<\infty$, where

$$
\left\|f_{\alpha}\right\|_{E}=\sup \left\{\left\|f_{\alpha} * f\right\|_{1}: f \in J(E),\|f\|_{1} \leqslant 1\right\}
$$

Received by the editors August 30, 1978 and, in revised form, December 14, 1978.

AMS (MOS) subject classifications (1970). Primary 43A45.

Key words and phrases. Hypergroup, ultra-strong Ditkin set, strong Ditkin set, compactly separating set.

(c) 1979 American Mathematical Society $0002-9939 / 79 / 0000-0561 / \$ 02.50$ 
and

(iii) for $f \in k(E), f * f_{\alpha} \rightarrow f$ in $L^{1}(K)$, where $J(E)$ is the closure of $\{f \in$ $L^{1}(K): \hat{f}$ vanishes in a neighborhood of $\left.E\right\}$ and $k(E)=\left\{f \in L^{1}(K): \hat{f}\right.$ vanishes on $E$ \}.

$E$ will be called ultra-strong Ditkin if it satisfies (i) and (iii) of above and $\sup \left\{\left\|f_{\alpha}\right\|_{1}: \alpha \in D\right\}<\infty$.

The empty set and the members of the center $Z(\hat{K})$ of $\hat{K}$ are all ultra-strong Ditkin, other points of $\hat{K}$ need not even be spectral. Nothing is known about the status of subhypergroups of $\hat{K}$. In this paper we introduce a class of closed subsets $E$ of $\hat{K}$, called compactly separating, for which $E$ is ultrastrong Ditkin whenever $\mathrm{Bd} E$ is. This class includes the classes of open subhypergroups of $\hat{K}$ whose complements are compact and of complements of compact open subhypergroups of $\hat{K}$. This immediately gives that all such hypergroups are ultra-strong Ditkin. We characterize this class (and also the ultra-strong Ditkin sets) for the example given by Dunkl and Ramirez [4] and further prove that not every closed subset is ultra-strong Ditkin even though every closed subset is strong Ditkin [4], [2], [1]. We shall freely use the notations, terminology and results on spectral synthesis for the hypergroup algebra $L^{1}(K)$ from [1], [2]. In particular, $K$ will be a commutative hypergroup whose dual $\hat{K}$ is a hypergroup and equals $\chi_{b}(K)$. We just remark that most of the results can be reformulated even when $\hat{K}$ is not $\chi_{b}(K)$ à la Theorem 3.3 [2]. Further their analogues for Segal algebras based on [1] can also be proved.

2. Definition 1. Let $E$ and $F$ be closed subsets of $\hat{K}$ and $\alpha>1$. $F$ will be said to be $\alpha$-boundedly disjoint from $E$ if there exists a symmetric neighborhood $V$ of 1 with compact closure such that

(i) $(F * V * V) \cap E=\Phi$ and

(ii) $\pi(F * V) / \pi(V)<\alpha$.

Definition 2. A closed subset $E$ of $\hat{K}$ will be called compactly separating if for some $\alpha>1$, every compact subset of $\hat{K} \backslash E$ is $\alpha$-boundedly disjoint from $E$.

LEMMA 3. Let $F$ be a compact subset of $\hat{K}$ which is $\alpha^{2}$-boundedly disjoint from a closed subset $E$ of $\hat{K}$. Then there exists $a \varphi \in A_{00}(\hat{K})$ such that $\varphi$ is 1 on $F$, zero on $E, 0<\varphi<1$ and $\|\varphi\|_{A}<\alpha$.

Proof. Choose $V$ as in Definition 1 and then apply Lemma 2.5 [2].

THEOREM 4. Let $E$ be a compactly separating subset of $\hat{K}$. If $\mathrm{Bd} E$ is ultra-strong Ditkin then so is $E$.

Proof. Because of Lemma 3, Rosenthal's proof of the corresponding result viz. Theorem 2.4(b)[9] for strong Ditkin sets can be modified to give this result. 
REMARK 5. We just note that ultra-strong Ditkin sets need not be compactly separating. For instance it can be easily seen that no point of the group $T$ is compactly separating.

COROllary 6. Let $E_{0}$ denote the set of points of $\hat{K}$ which are ultra-strong Ditkin. If $\hat{K}$ is discrete at points of $\hat{K} \backslash F_{0}$ for some finite subset $F_{0}$ of $E_{0}$ then every compactly separating subset of $\hat{K}$ is ultra-strong Ditkin.

Proof. It follows immediately from the above theorem since finite unions of ultra-strong Ditkin sets are ultra-strong Ditkin [1, Remark 3.4(vi)].

REMARK 7. The corresponding results for Calderon ( $F_{0}$ can be even countable) and strong Ditkin sets can be easily formulated.

THEOREM 8. Let $H$ be an open subhypergroup of $\hat{K}$.

(i) if $H$ is compact then $\hat{K} \backslash H$ is compactly separating,

(ii) if $\hat{K} \backslash H$ is compact then $H$ is compactly separating.

Proof. (i) Take $V=H$. For any compact subset $F$ of $H, F * V * V=$ $F * V \subset H$ and thus $F$ is 1-boundedly disjoint from $\hat{K} \backslash H$.

(ii) Let $V$ be any compact neighborhood of 1 contained in $H$. By $[7,10.3 \mathrm{~A}]$ $(\hat{K} \backslash H) * H * H=\hat{K} \backslash H$.

Let $\alpha=\pi(\hat{K} \backslash H) / \pi(V)$. So for any compact subset $F$ of $\hat{K} \backslash H$, $(F * V * V) \cap H=\Phi$ and $\pi(F * V) / \pi(V)<\alpha$. Thus $F$ is $\alpha$-boundedly disjoint from $H$.

THEOREM 9. (i) The complement of a compact open subhypergroup $H$ in $\hat{K}$ is ultra-strong Ditkin.

(ii) An open subhypergroup with a compact complement is ultra-strong Ditkin.

Proof. The sets in both the cases are closed and have empty boundary. Further $\Phi$ is ultra-strong Ditkin by Remark 3.4(ii) [1], which in fact, follows immediately from Theorem 2.8 of [2] on the existence of a bounded approximate identity in $L^{1}(K)$.

EXAMPLE 10. Let $p$ be a prime number and $a=1 / p$. Then the hypergroup $H_{a}$ is defined by Dunkl and Ramirez [4] to be the one point compactification $Z_{+}^{*}$ of the set $Z_{+}$of positive integers with Haar measure $m$ given by

$$
\begin{aligned}
m(k) & =(1-a) a^{k}, \quad k \neq \infty, \\
& =0, \quad k=\infty,
\end{aligned}
$$

and convolution given by

$$
\begin{gathered}
p_{n} * p_{m}=p_{\min (n, m)} \quad \text { for } n, m \in Z_{+}^{*} \text { and } n \neq m, \text { for } n \in Z_{+}, \\
p_{n} * p_{n}(t)= \begin{cases}0, & t<n, \\
\frac{1-2 a}{1-a}, & t=n, \\
a^{k}, & t=n+k>n,\end{cases}
\end{gathered}
$$

and $p_{\infty} * p_{\infty}=p_{\infty}$. 
As explained in Example 4.6 [2] $H_{a}$ can be considered as $\hat{K}$, where $K$ is $\hat{H}_{a}$ and members of $\hat{H}_{a}$ are given by $\left\{\chi_{n}: n \in Z_{+}\right\}$where

$$
\chi_{n}(k)= \begin{cases}0, & k<n-1, \\ a /(a-1), & k=n-1, \\ 1, & k>n \text { or } k=\infty .\end{cases}
$$

(i) A closed subset $E$ of $\hat{K}$ is compactly separating if and only if either $E$ is finite and $\infty \notin E$ or $\hat{K} \backslash E$ is finite if and only if $E$ is open.

(ii)(a) All closed open subsets of $\hat{K}$ are ultra-strong Ditkin.

(b) All finite subsets of $\hat{K}$ are ultra-strong Ditkin.

(c) An infinite closed subset of $\hat{K}$ whose complement is infinite is not ultra-strong Ditkin.

Equivalently,

(ii)' A closed subset $E$ of $\hat{K}$ is ultra-strong Ditkin if and only if it is finite or open.

Proof. (i)(a) Let $E \subset \hat{K}$ be closed such that $\hat{K} \backslash E$ is finite. Then $\infty \in E$.

Let $k_{0}=\max \{k: k \in \hat{K} \backslash E\}, V=\left\{k \in \mathbf{N}: k>k_{0}\right\} \cup\{\infty\}$ and $\alpha=$ $1 / m(V)$. Then for any compact subset $F$ of $\hat{K} \backslash E, F * V * V=F * V=F$, also $m(F)<1$ and therefore, $F$ is $\alpha$-boundedly disjoint from $E$. Hence $E$ is compactly separating.

(b) Let $E \subset \hat{K}$ be finite and $\infty \notin E$. Let $k_{0}=\max \{k: k \in E\}, V=\{k \in$ $\left.\mathbf{N}: k>k_{0}\right\} \cup\{\infty\}$ and $\alpha=1 / m(V)$. Let $B=\left\{k \in Z_{+}: k \leqslant k_{0}\right\} \backslash E$. Then $\hat{K} \backslash E=V \cup B$. So

$$
(\hat{K} \backslash E) * V * V=(V \cup B) * V * V=V \cup B=\hat{K} \backslash E .
$$

So for any compact subset $F$ of $\hat{K} \backslash E,(F * V * V) \cap E=\Phi$ and $m(F * V) / m(V) \leqslant \alpha$. Hence $E$ is compactly separating.

(c) Let $E$ be a closed subset of $\hat{K}$ such that it is not of any of the above two types.

Then $\hat{K} \backslash E$ is infinite and $\infty \in E$. Let $\hat{K} \backslash E=\left\{k_{i}: i \in \mathbf{N}\right\}$, where $\left\{k_{i}\right\}$ is a strictly increasing sequence of positive integers. Let, if possible, $E$ be compactly separating and $\alpha$ a bound. Then $F_{j}=\left\{k_{i}: 1 \leqslant i \leqslant j\right\}$ is a compact subset of $\hat{K} \backslash E$ and therefore, there exists a (symmetric) neighborhood $V_{j}$ of 1 such that $\left(F_{j} * V_{j} * V_{j}\right) \cap E=\Phi$ and $m\left(F_{j} * V_{j}\right) / m\left(V_{j}\right) \leqslant \alpha$. If $V_{j} \not \subset\{k$ : $\left.k>k_{j}\right\}$ then there is a $p \leqslant k_{j}$ such that $p \in V_{j}$. There are two possibilities

(i) $p \in F_{j} \cap V_{j}$ : Then

$$
\{k \in \mathbf{N}: k>p\} \subset p * p \subset F_{j} * V_{j} \subset F_{j} * V_{j} * V_{j}
$$

But $E$ is infinite so $\{k \in \mathbf{N}: k>p\} \cap E \neq \Phi$, which contradicts $\left(F_{j} * V_{j} * V_{j}\right) \cap E=\Phi$.

(ii) $p \in V_{j} \backslash F_{j}$, then $p \in E$. Also $p \in k_{j} * p \subset F_{j} * V_{j}$. So $p \in F_{j} * V_{j} * V_{j}$, which is a contradiction. Hence $V_{j} \subset\left\{k: k>k_{j}\right\}$. So $m\left(V_{j}\right)$ is less than or equal to $a^{k_{j}+1}$. Also $F_{j} * V_{j}=F_{j}$. So

$$
m\left(F_{j} * V_{j}\right)=m\left(F_{j}\right)=\sum_{i=1}^{j} a^{k_{i}}(1-a) \geqslant j a^{k_{j}}(1-a) .
$$


So $m\left(F_{j} * V_{j}\right) / m\left(V_{j}\right)>j a^{k_{j}}(1-a) / a^{k_{j}+1}=j(1-a) / a$.

So $j<\alpha a /(1-a)$, a contradiction. Hence $E$ is not compactly separating subset of $\hat{K}$.

(d) The last equivalence now follows immediately because a closed set $E$ in $\hat{K}$ is open if and only if $E$ is finite and $\infty \notin E$ or $\hat{K} \backslash E$ is finite and $\infty \in E$.

(ii)(a) Follows from (i) and Theorem 5 above.

(b) If $E$ is finite and $\infty \notin E$ then $E$ is ultra-strong Ditkin by (i). If $E$ is finite and $\infty \in E$ then $E \backslash\{\infty\}$ is ultra-strong Ditkin by the above argument. Also $\{\infty\}$ is ultra-strong Ditkin by Theorem 3.3 [2] and therefore, by Remark 3.4(vi) [1], $E$ is ultra-strong Ditkin.

(c) Let, if possible, $E$ be ultra-strong Ditkin, since $E$ is infinite and $\hat{K} \backslash E$ is infinite there exists a sequence $\left\{\alpha_{n}\right\}$ in $\mathbf{N}$ such that $\alpha_{n}<\alpha_{n}+1<\alpha_{n+1}$, $\alpha_{n} \in \hat{K} \backslash E$ and $\alpha_{n}+1 \in E$ for all $n$. Define $\varphi$ on $\hat{K}$ by $\varphi\left(\alpha_{n}\right)=1 / n^{2}$ and zero otherwise. Then $\varphi \in A(\hat{K})$ by Theorem 7.8 [4]. Further $\varphi=0$ on $E$. Let $\varphi=\hat{f}$ for $f \in L^{1}(K)$ then $f \in k(E)$. Since $E$ is ultra-strong Ditkin, $k(E)$ has factorization by Cohen's factorization theorem. So there exists $g, h \in k(E)$ such that $f=g * h$.

Then $\hat{g}\left(\alpha_{n}+1\right)=0$ for each $n$. So by Theorem 7.8 [4]

$$
\sum\left|\hat{g}\left(\alpha_{n}\right)\right| \leqslant \sum|\hat{g}(n)-\hat{g}(n-1)|<\infty .
$$

Similarly $\Sigma\left|\hat{h}\left(\alpha_{n}\right)\right|<\infty$. Also $1 / n^{2}=\hat{f}\left(\alpha_{n}\right)=\hat{g}\left(\alpha_{n}\right) \hat{h}\left(\alpha_{n}\right)$.

So by the Cauchy-Schwarz inequality

$$
\begin{aligned}
\sum \frac{1}{n} & =\sum \sqrt{\left|\hat{g}\left(\alpha_{n}\right)\right|} \sqrt{\left|\hat{h}\left(\alpha_{n}\right)\right|} \\
& \leqslant\left(\sum\left|\hat{g}\left(\alpha_{n}\right)\right|\right)^{1 / 2}\left(\sum\left|\hat{h}\left(\alpha_{n}\right)\right|\right)^{1 / 2} \\
& <\infty, \text { a contradiction. }
\end{aligned}
$$

Hence $E$ is not ultra-strong Ditkin.

We thank the referee for his useful comments and suggestions.

\section{REFERENCES}

1. A. K. Chilana and A. Kumar, Spectral synthesis in Segal algebras on hypergroups, Pacific J. Math. 79 (1978).

2. A. K. Chilana and K. A. Ross, Spectral synthesis in hypergroups, Pacific J. Math. 76 (1978), 313-328.

3. C. F. Dunkl, The measure algebra of a locally compact hypergroup, Trans. Amer. Math. Soc. 179 (1973), 331-348.

4. C. F. Dunkl and D. E. Ramirez, $A$ family of countable compact $\boldsymbol{P}_{*}$-hypergroups, Trans. Amer. Math. Soc. 202 (1975), 339-356.

5. J. E. Gilbert, On projections of $L^{\infty}(G)$ onto translation-invariant subspaces, Proc. London Math. Soc. 19 (1969), 69-88.

6. E. Hewitt and K. A. Ross, Abstract harmonic analysis. I, II, Springer-Verlag, Berlin and New York, 1963, 1970.

7. R. I. Jewett, Spaces with an abstract convolution of measures, Advances in Math. 18 (1975), $1-101$.

8. T. S. Liu, A. V. Rooij and J. K. Wang, Projections and approximate identities for ideals in group algebras, Trans. Amer. Math. Soc. 175 (1973), 469-482. 
9. H. P. Rosenthal, On the existence of approximate identities in ideals of group algebras, Ark. Mat. 7 (1967), 185-191.

10. B. M. Schreiber, On the coset ring and strong Ditkin sets, Pacific J. Math. 32 (1970), 805-812.

11. R. Spector, Apercu de la théorie des hypergroupes, Lecture Notes in Math., vol. 497, (Analyse Harmonique sur les Groupes de Lie, Sem. Nancy-Strasbourg 1973-1975), SpringerVerlag, New York, 1975.

12. __, Mesures invariantes sur les hypergroupes, Trans. Amer. Math. Soc. 239 (1978), 147-166.

13. I. Wik, A strong form of spectral synthesis, Ark. Mat. 6 (1965), 55-64.

Department of Mathematics, University of Delhi, Delhn-110007, India 